\title{
Effets d'amendements organiques et phosphatés sous zaï sur les propriétés chimiques et biologiques du sol et la qualité de la matière organique en zone soudano-sahélienne du Burkina Faso
}

\author{
Abdoulaye DABRE ${ }^{1}$, Edmond $\mathrm{HIEN}^{2 *}$, Der $\mathrm{SOME}^{2}$ et Jean Jacques Drevon ${ }^{3}$ \\ ${ }^{1}$ Bureau National des Sols, E-mail : dabreab@gmail.com, 03 BP 7142 Ouagadougou 03 Burkina Faso. \\ ${ }^{2}$ Université Ouaga I Pr Joseph KI-ZERBO, UFR/SVT \& IRD, LMI-IESOL ; 03 BP 7021, Ouagadougou 03. \\ ${ }^{2}$ Université Ouaga I Pr Joseph KI-ZERBO, UFR/SVT \& IRD, LMI-IESOL 03 BP 7021, Ouagadougou 03, \\ Burkina Faso. \\ ${ }^{3}$ UMR Eco\&Sols; Ecologie Fonctionnelle \&. Biogéochimie des Sols \& Agroécosystèmes, E-mail : \\ drevonjj@supagro.inra.fr, 2 Place Viala,F34060, Montpellier. France. \\ Auteur correspondant, E-mail : edmond.hien@ird.fr
}

\section{REMERCIEMENTS}

Les auteurs remercient le Projet FABATROPIMED de la Fondation Agropolis International (France) qui a soutenu financièrement la réalisation de ces travaux. Ils remercient sincèrement l'UMR 210 Eco\&Sols (Ecologie Fonctionnelle \& Biogéochimie des sols \& des Agroécosystèmes) de l'IRD pour son soutien matériel et financier.

\section{RESUME}

Le zaï est une pratique d'adaptation utilisée sur les sols dégradés souvent encroutés (zipella) en Afrique sub-sahélienne pour pallier l'aridité climatique dans un contexte de changements globaux. L'objectif de cette étude est de proposer une pratique culturale adaptée à la restauration des zipella en mesurant les effets de fumures organique et phosphatée sous zaï. Un essai conduit à Pougyango, au Nord du Burkina Faso a permis de tester 5 traitements comprenant ou non des apports organique et phosphaté sous zaï. Les paramètres mesurés portent sur le carbone, l'azote total, le $\mathrm{pH}$, la respiration du sol et la qualité de matière organique du sol dans les poquets et dans les interpoquets. Les résultats montrent que le zaï améliore les teneurs en carbone $(1,22 \%$ sous niébé ; $1,18 \%$ sous sorgho). Le niébé améliore significativement la teneur de l'azote $(0,094 \% \mathrm{~s}$ contre $0,080 \%$ sous sorgho). Le dégagement de $\mathrm{CO}_{2}$ atteint de $9,25 \mathrm{~g} \cdot \mathrm{kg}^{-1}$ de sol dans le traitement sous zaï associant le fumier et le phosphate contre $2,06 \mathrm{~g} \cdot \mathrm{kg}^{-1}$ dans le témoin sous sorgho. Le carbone et l'azote du sol sont préférentiellement stockés dans les fractions fines. Ainsi, la fraction 0-20 $\mu \mathrm{m}$ stocke 65,53 et $66,53 \%$ de l'azote du sol respectivement sous niébé et sous sorgho.

(C) 2016 International Formulae Group. All rights reserved.

Mots clés : Zaï, fumures organo-minérales, carbone, azote, respiration du sol.

\section{Effects of organic and phosphate amendments in zaï system on soil chemical and biological properties and quality of organic matter in the Sudano- Sahelian zone of Burkina Faso}

\begin{abstract}
Zaï is an adaptive farming practice used on degraded soils often encrusted (zipella) in sub-Saharan Africa, to address the climate aridity in a context of global changes. The objective of this study was to provide a cultural practice adapted for the restoration of zipella by measuring the effects of organic and phosphate
\end{abstract}


fertilizers in zaï system. A trial was set in Pougyango, a village located in northern Burkina Faso to test five treatments with or without organic and phosphate inputs under zai. The parameters measured were carbon, total nitrogen, $\mathrm{pH}$, soil respiration and quality of soil organic matter in zaï holes or out of holes. The results showed that zai improves the carbon content (1.22\% under cowpea, and $1.18 \%$ under sorghum). Cowpea significantly improves nitrogen content $\left(0.094 \%\right.$ vs $0.080 \% \mathrm{~s}$ in sorghum). The release of $\mathrm{CO}^{2}$ reached $9.25{\mathrm{~g} . \mathrm{kg}^{-1}}$ soil in the treatment under zaï combining manure and phosphate vs $2.06 \mathrm{~g} \cdot \mathrm{kg}^{-1}$ in the control with sorghum. Carbon and nitrogen from the soil are preferably stored in the fine fractions. Thus, the fraction 0-20 microns stores 65.53 and $66.53 \%$ of soil nitrogen respectively with cowpea or sorghum.

(C) 2016 International Formulae Group. All rights reserved.

Keywords: Zaï, organo-mineral manures, carbon, nitrogen, soil respiration.

\section{INTRODUCTION}

La dégradation des sols est un problème majeur de l'agriculture burkinabè. Elle est corrélée avec les conditions pédoclimatiques de plus en plus défavorables, la poussée démographique qui limite voire supprime la jachère et les mauvaises pratiques comprenant la culture continue de céréales sans apport de fertilisants (Sawadogo, 2006 ; MARH, 2008). En effet, la dégradation affecte de nos jours plus de $24 \%$ des terres arables au Burkina. D'autres études estiment que les terres sont à des niveaux de dégradation très élevé $(11 \%)$, élevé $(2 \%)$, moyen $(34 \%)$ et faible (49\%). Traoré et Toé (2008) soulignent que les sols du Burkina Faso sont naturellement pauvres en matières organiques notamment en éléments nutritifs essentiels dont l'azote et le phosphore. Tous ces facteurs ont concouru à la formation des sols nus appelés zipella (clairière ou zone blanche en langue mooré) (Zombré, 2003), hardés au Cameroun, wala-wala au Mali et gangani au Niger (Mando et al., 2001). Un des grands défis de notre agriculture consisterait à maîtriser le processus de récupération et à limiter l'influence des facteurs de formation de ces sols nus dans un contexte de changements globaux. Des actions orientées vers des systèmes de cultures plus durables (pratiques culturales adaptées, emploi conséquent de la fumure organique et capitalisation de l'activité biologique surtout celle des rhizobia et des champignons mycorhiziens) peuvent être entreprises pour faire face à la dégradation. C'est la raison pour laquelle, les populations ont développé des technologies de conservation des eaux et des sols puis la défense et la restauration des sols comme le zaï et les associations céréaleslégumineuses (Zombré, 2003 ; Somé et al., 2004 ; Barro et al., 2005 ; Sawadogo et al., 2008) pour s'adapter aux irrégularités pluviométriques. C'est une technique ancestrale pour restaurer la productivité des terres agricoles dégradées. Les pratiques paysannes comportant des associations ou des rotations légumineuses-céréales ont déjà fait l'objet d'études pour appréhender leur efficacité sur la productivité (Dabat, 2011; Zeinabou et al., 2014; Ouandaogo et al., 2016). La culture des légumineuses a un rôle bénéfique sur la fertilité du sol et les rendements des céréales subséquentes (Jemo et al., 2006). Les quantités d'azote fixé par du niébé en milieu paysan (Naab et al., 2009) ont permis l'obtention des résultats variables en fonction des facteurs principaux tels les variétés cultivées, les fertilisations pratiquées et les champs paysans étudiés (Traoré, 2012). La présente étude se propose d'évaluer les effets du zaï sur les propriétés chimiques et biologiques des sols et la qualité de la matière organique sous culture de niébé et de sorgho. Elle se propose en plus d'apporter un éclairage sur les impacts combinés du zaï et d'une céréale ou d'une légumineuse sur la répartition de la matière organique. 


\section{MATERIELS ET METHODES}

\section{Site d'étude}

L'essai agronomique a été mis en place à Pougyango, un village situé dans le département de Gomponsom, province du Passoré sur l'axe Yako-Kaya à $10 \mathrm{~km}$ au Nord-Est de Yako. Les coordonnées géographiques de la zone d'étude sont les suivantes : $12^{\circ} 58^{\prime}$ de longitude, $2^{0} 08^{\prime}$ de latitude et de $307 \mathrm{~m}$ d'altitude.

\section{Matériels}

Le sol utilisé est un sol ferrugineux tropical lessivé induré superficiel (CPCS, 1967) ou leptosol pétroférique selon la World Reference Base (FAO, 2014). C'est un sol totalement nu appelé zipellé. Les échantillons de sol ont été prélevés à la fin de la campagne agricole 2012 (novembre) à la récolte dans la couche $0-20 \mathrm{~cm}$. Les prélèvements élémentaires de sol ont été faits sur la diagonale en trois points. Ces échantillons ont été ensuite séchés, tamisés, puis broyés pour les analyses.

Les espèces végétales utilisées sont le sorgho (Sorghum bicolor L; variété ICSV 1049) et le niébé (Vigna unguiculata (L.) Walp., variété KVX 61-1).

Le fumier utilisé est un fumier de parc (caprins et bovins). Le compost est produit en milieu paysan à partir du compostage des ordures ménagères et de résidus de récolte issu d'une fosse compostière. Le Burkina phosphate (BP) utilisé est un phosphate naturel. Les caractéristiques chimiques des amendements sont résumées dans le Tableau 1.

\section{Dispositif expérimental}

Le dispositif utilisé est un split plot à deux facteurs prenant en compte le facteur amendement et le facteur matériel végétal. L'essai comprend trois blocs (répétitions) distants de $5 \mathrm{~m}$. Chaque bloc comporte 12 parcelles dont 6 parcelles cultivées en niébé et 6 en sorgho avec des allées de $1 \mathrm{~m}$ entre les parcelles dans un même bloc. Chaque parcelle a une superficie unitaire de $20 \mathrm{~m}^{2}$. Dans chaque parcelle, 99 poquets de zaï sont creusés selon un rayonnage croisé, avec un diamètre d'environ $25 \mathrm{~cm}$ et une profondeur de 10 à $15 \mathrm{~cm}$. Une diguette antiérosive formée de cordon pierreux a été aménagée en amont de l'essai afin de ralentir le ruissellement.

Les six traitements appliqués étaient les suivants :

TA : Témoin absolu; ZS : Zaï simple; ZC : $Z a \ddot{l}+$ compost à 3 t.ha ${ }^{-1}$ (soit $61 \mathrm{~g} /$ poquet) ; $\mathrm{ZF}$ : Zaï + fumier à $3 \mathrm{t} \cdot \mathrm{ha}^{-1}$ (soit $61 \mathrm{~g} /$ poquet) ; ZCP : Zaï +compost à 3t.ha ${ }^{-1}$ (soit $61 \mathrm{~g} /$ poquet $)+$ Burkina phosphate (BP) à 2t.ha ${ }^{-}$ ${ }^{1}$ (soit 40,5g/poquet); ZFP : Zaï + fumier à 3 t.ha $^{-1}$ (soit $61 \mathrm{~g} /$ poquet) + Burkina phosphate (BP) à $2 \mathrm{t} \mathrm{ha}^{-1}$ (soit 40,5g/poquet).

\section{Paramètres mesurés}

Au laboratoire de Pédologie de l'IRD de Ouagadougou au Burkina Faso, le pHeau a été mesuré par électrométrie à l'aide de $\mathrm{pH}$ mètre de marque HANNA dans une suspension sol/eau (de rapport 2/5) après mise en agitation du sol avec de l'eau déminéralisée durant une (1) heure. L'activité biologique potentielle $\left(\mathrm{C}-\mathrm{CO}_{2}\right.$ dégagé $)$ du sol prélevé dans les poquets de zaï a été évaluée par la méthode du test respirométrique à l'aide d'un respiromètre «DIMARSOL». Pour cela, $2 \mathrm{~g}$ de chaque échantillon de sol issu des 36 poquets de zaï du site ont été mis dans un tube anticoagulant en verre (3 répétitions) et humidifiés avec $700 \mu \mathrm{l}$ d'eau distillée. Les tubes fermés hermétiquement ont été ensuite incubés dans l'obscurité à la température ambiante jusqu'à la période de lecture. La première lecture du dégagement de $\mathrm{CO}_{2}$ a été faite après 4 heures d'incubation; les autres lectures ont été réalisées par intervalle de 96 heures d'incubation de la lecture précédente et cela, pendant 2 semaines.

Le fractionnement granulométrique de la matière organique a concerné les échantillons 
de sols prélevés dans les poquets des traitements TA, ZS, ZF et ZFP. La méthode utilisée est celle décrite par Feller (1979). Les fractions considérées ont été les suivantes :

Fraction A : $0-20 \mu \mathrm{m}$; fraction B : 20-50 $\mu \mathrm{m}$; fraction $\mathrm{C}: 50-2000 \mu \mathrm{m}$.

Le carbone $(\mathrm{C})$ et l'azote $(\mathrm{N})$ de ces fractions et des 66 échantillons de sols provenant du site agronomique de Pougyango ont été dosés au CHN au laboratoire des moyens analytiques de l'IRD de Dakar au Sénégal.

\section{Traitement des données}

Les analyses statistiques ont été réalisées par le logiciel XLSTATS version 13. Les procédures utilisées ont été l'analyse de la variance (ANOVA) et la séparation des moyennes par le test de Student NewmanKeuls (SNK) au seuil de 5\%. Les histogrammes et les courbes ont été construits avec le tableur Excel 2010.

\section{RESULTATS}

\section{Effets des traitements sur le $\mathbf{p H}$ du sol}

Les résultats présentés dans le Tableau 2 indiquent que les $\mathrm{pH}$ obtenus dans les poquets $(5,71$ pour le niébé ; 5,78 pour le sorgho) sont significativement plus élevés que ceux enregistrés sur les interpoquets $(5,44$ pour le niébé ; 5,54 pour le sorgho) sous les deux cultures. Dans les poquets ensemencés avec le niébé, le ZCP et le ZF ont significativement occasionné une baisse d'acidité évaluée à $8,05 \%$ et à $20,08 \%$ par rapport respectivement au ZS et au TA. La réalisation du zaï a statistiquement permis le relèvement de l'acidité du zipellé qu'il soit associé ou non à un apport de la matière organique. Sur les interpoquets, les valeurs de $\mathrm{pH}$ ont constitué un groupe homogène avec une valeur maximale de 5,58 notée dans les traitements ZC et ZFP contre 5,03 pour le témoin. Dans les poquets de zaï semés au sorgho, le ZFP et le ZCP ont entrainé un plus grand relèvement de $\mathrm{pH}$ estimé respectivement à $9,12 \%$ et $8,76 \%$ par rapport au ZS puis à $22,98 \%$ et $22,58 \%$ par rapport au TA. Sur leurs interpoquets, le zaï a favorisé une baisse significative de l'acidité par rapport au témoin absolu. Aussi, le relèvement supérieur de l'acidité du sol constaté sous sorgho $(5,66)$ n'a pas été significatif par rapport à celui enregistré sous niébé $(5,57)$.

\section{Effets des pratiques sur la teneur en carbone du sol}

Les résultats obtenus (Tableau 3) montrent que le taux de carbone des interpoquets $(1,11 \%$ sous niébé ; $1,07 \%$ sous sorgho) est statistiquement inférieur à celui des poquets $(1,22 \%$ sous niébé ; $1,18 \%$ sous sorgho) qu'ils soient ensemencés avec le niébé ou le sorgho. Dans les poquets où le niébé a été semé, les traitements ZF (1,38\%) et ZFP $(1,34 \%)$ ont présenté des teneurs en carbone statistiquement plus importantes que sous ZS (1,01\%). Les teneurs en carbone des traitements ZCP $(1,21 \%), \mathrm{ZC}(1,20 \%)$ et TA $(1,19 \%)$ sont moyennes par rapport à l'ensemble des traitements. Dans les poquets de zaï semés avec le sorgho, le traitement ZF $(1,47 \%)$ a permis une évolution significative de la teneur en carbone par rapport au TA $(1,04 \%)$ et au ZS $(0,93 \%)$. De façon générale, il n'y a pas eu une différence significative au niveau des interpoquets et entre les cultures ( $1,12 \%$ sous niébé $; 1,17 \%$ sous sorgho). Les poquets de zaï n'ayant pas bénéficié d'un apport de matière organique ont induit une teneur inférieure en carbone comparativement au témoin.

\section{Effets des pratiques sur la teneur en azote du sol}

Les teneurs en azote total des sols sont résumées dans le Tableau 4. Il ressort de l'analyse de variance qu'au niveau des interpoquets, les différences entre les traitements ne sont pas statistiquement significatives sous niébé $(0,080 \%)$ tout comme sous sorgho $(0,074 \%)$. Dans les poquets sous niébé, deux groupes 
statistiquement différents ont été distingués : le groupe homogène composé de ZF $(0,110 \%)$, ZFP $(0,105 \%)$, ZC $(0,093 \%)$, ZCP $(0,092 \%)$ et TA $(0,092 \%)$ d'une part et celui de ZS $(0,070 \%)$ d'autre part. Quant aux poquets dans lesquels le sorgho a été semé, le taux d'azote du ZF $(0,111 \%)$ a été significativement supérieur à ceux de ZCP $(0,082 \%), \mathrm{ZC}(0,081 \%)$ et TA $(0,080 \%)$ qui ont été aussi statistiquement supérieur à celui ZS $(0,065 \%)$. De plus, les valeurs observées dans les poquets sont significativement différentes de celles des interpoquets sous les deux cultures. Le niébé $(0,087 \%)$ a permis une évolution significative du taux d'azote par rapport au sorgho $(0,080 \%)$. Les traitements ayant bénéficié de l'apport du Burkina phosphate (ZFP et ZCP) ont un taux d'azote légèrement faible par rapport à $\mathrm{ZF}$ et $\mathrm{ZC}$. De même, les poquets de zaï sans apport du compost et de fumier ont un taux d'azote inférieur à celui du témoin.

\section{Effets des pratiques sur la répartition de la matière organique du sol}

Les résultats $\mathrm{du}$ fractionnement granulométrique de la matière organique sont consignés dans le Tableau 5. Les bilans de masse du poids total de l'échantillon fractionné par rapport à la somme des poids des fractions obtenues sont compris en moyenne entre 100 et $101 \%$. Ces valeurs se situant dans la gamme de 99 à $102 \%$ attestent donc la fiabilité des résultats obtenus à la suite $\mathrm{du}$ fractionnement de la matière organique. Les teneurs en $\mathrm{C}$ et $\mathrm{N}$ des fractions sont présentées dans le Tableau 6. Il ressort que le taux d'azote a été plus élevé dans la fraction $\mathrm{A}$ $\left(2,11\right.$ g. $\left.\mathrm{kg}^{-1}\right)$ que ceux de la fraction C $(0,81$ g.kg $\left.{ }^{-1}\right)$ et de la fraction $B\left(0,30\right.$ g. $\left.\mathrm{kg}^{-1}\right)$ sous niébé. Il a été plus élevé au niveau du traitement ZFP (1,24 g.kg $\left.{ }^{-1}\right)$ comparativement au ZF $\left(1,22\right.$ g.kg $\left.{ }^{-1}\right)$, au TA $\left(0,94\right.$ g.kg $\left.{ }^{-1}\right)$ et au ZS $\left(0,87\right.$ g. $\left.\mathrm{kg}^{-1}\right)$. La teneur en carbone est de $19,78 \mathrm{~g} \cdot \mathrm{kg}^{-1}$ dans la fraction A, de $11,36 \mathrm{~g} \cdot \mathrm{kg}^{-1}$ dans la fraction $\mathrm{C}$ et de $4,53 \mathrm{~g} \cdot \mathrm{kg}^{-1}$ dans la fraction $\mathrm{B}$ sous niébé. Le rapport $\mathrm{C} / \mathrm{N}$ décroit de la fraction grossière à la fraction fine. Il est de 15,5 dans la fraction $C$, de 15,4 dans la fraction B et de 9,4 dans la fraction A sous niébé. Sous sorgho, la même tendance a été observée dans les différentes fractions avec des valeurs de $\mathrm{C}$ et de $\mathrm{N}$ relativement faibles par rapport à celles obtenues sous niébé. La teneur en azote la plus élevée a été obtenue dans la fraction A $\left(2,02\right.$ g. $\left.\mathrm{kg}^{-1}\right)$ par rapport à celles notées dans les fractions $\mathrm{C}\left(0,74 \mathrm{~g} \cdot \mathrm{kg}^{-1}\right)$ et $\mathrm{B}\left(0,28 \mathrm{~g} \cdot \mathrm{kg}^{-1}\right)$. Le taux d'azote est plus élevé dans le traitement ZF (1,25 g. $\left.\mathrm{kg}^{-1}\right)$ par rapport au ZFP (1,14 g. $\left.\mathrm{kg}^{-1}\right)$, au TA $(0,84$ g.kg $\left.{ }^{-1}\right)$ et au ZS $\left(0,80\right.$ g.kg $\left.{ }^{-1}\right)$. Le rapport $\mathrm{C} / \mathrm{N}$ est de 17 dans la fraction $\mathrm{C}, 14,8$ dans la fraction $\mathrm{B}$ et de 9,8 dans la fraction $\mathrm{A}$.

\section{Effets des pratiques sur le dégagement de $\mathrm{CO}_{2}$ du sol}

Les résultats révèlent que le dégagement journalier de $\mathrm{CO}_{2}$ croit suivant le temps d'incubation (Figures 1 et 2). Sous sorgho (Figure 1), les dégagements cumulés de $\mathrm{CO}_{2}$ ont été plus élevés au niveau du sol aménagé en zaï associé ou non à l'apport des amendements comparativement au témoin. Les valeurs cumulées les plus importantes sont notées dans le ZFP $\left(9,25\right.$ g. $\mathrm{kg}^{-1}$ de sol) par rapport au témoin $\left(2,06 \mathrm{~g} \cdot \mathrm{kg}^{-1} \mathrm{de}\right.$ sol). Les traitements ZCP $\left(5,79\right.$ g. $\mathrm{kg}^{-1}$ de sol), ZF (5,79 g. $\mathrm{kg}^{-1}$ de sol), ZC $\left(5,74\right.$ g. $\mathrm{kg}^{-1}$ de sol) et ZS $\left(3,83\right.$ g. $\mathrm{kg}^{-1}$ de sol) ont enregistré des dégagements cumulés de $\mathrm{CO}_{2}$ supérieurs au témoin absolu. Sous niébé (Figure 2), le cumul du dégagement journalier de $\mathrm{CO}_{2}$ durant toute la période de mesure avec un pic de 7,85 g. $\mathrm{kg}^{-1}$ dans le traitement ZFP. Les autres traitements ont enregistré respectivement des valeurs non moins importantes de cumuls de 6,24 g. $\mathrm{kg}^{-1}$ de sol (ZCP), de 5,22 g.kg-1 de sol (ZC), de 5,20 g.kg-1 de sol (ZF) et de 4,46 g.kg ${ }^{-1}$ de sol (ZS) par rapport à celui du témoin $\left(2,78 \mathrm{~g} \cdot \mathrm{kg}^{-1} \mathrm{de}\right.$ sol). En général, le dégagement journalier cumulé du $\mathrm{CO}_{2}$ a été plus élevé sous sorgho $\left(5,44 \mathrm{~g} \cdot \mathrm{kg}^{-1}\right.$ de sol $)$ que sous niébé $\left(5,29 \mathrm{~g} \cdot \mathrm{kg}^{-1}\right.$ de sol). 
Tableau 1 : Caractéristiques chimiques des amendements utilisés.

\begin{tabular}{|c|c|c|c|c|c|c|c|}
\hline Amendements utilisés & $\mathbf{p H}$ & $\mathrm{N}$ total (\%) & $\begin{array}{c}\text { C total } \\
(\%)\end{array}$ & $\begin{array}{c}\text { P total } \\
\left(\% P_{2} 0_{5}\right)\end{array}$ & $\begin{array}{c}\mathrm{CaO} \\
(\%)\end{array}$ & $\begin{array}{l}\mathrm{K} \text { total } \\
\left(\% \mathrm{~K}_{2} \mathrm{O}\right)\end{array}$ & $\mathbf{C} / \mathbf{N}$ \\
\hline Fumier & 8,6 & 1,35 & 14,89 & - & - & - & 11 \\
\hline Compost & 7,7 & 0,76 & 10,28 & - & - & - & 13,6 \\
\hline BP & & & 0,03 & 25,43 & 10,61 & 0,3 & - \\
\hline
\end{tabular}

Tableau $2: \mathrm{pH}_{\text {eau }}$ du sol.

\begin{tabular}{lcccc}
\hline \multirow{2}{*}{ Traitements } & \multicolumn{3}{c}{ Niébé } & \multicolumn{2}{c}{ Sorgho } \\
\cline { 2 - 5 } TH Poquets & $\mathbf{p H}$ Interpoquets & $\mathbf{p H}$ Poquets & pH Interpoquets \\
\hline $\mathbf{Z C}$ & $5,03 \pm 0,43 \mathbf{b}$ & $5,03 \pm 0,43 \mathbf{a}$ & $4,96 \pm 0,26 \mathbf{c}$ & $4,96 \pm 0,26 \mathbf{b}$ \\
$\mathbf{Z C P}$ & $5,64 \pm 0,35 \mathbf{a}$ & $5,58 \pm 0,34 \mathbf{a}$ & $5,93 \pm 0,26 \mathbf{a}$ & $5,79 \pm 0,27 \mathbf{a}$ \\
$\mathbf{Z F}$ & $6,04 \pm 0,10 \mathbf{a}$ & $5,48 \pm 0,24 \mathbf{a}$ & $6,08 \pm 0,20 \mathbf{a}$ & $5,58 \pm 0,16 \mathbf{a}$ \\
$\mathbf{Z F P}$ & $6,04 \pm 0,15 \mathbf{a}$ & $5,55 \pm 0,57 \mathbf{a}$ & $6,03 \pm 0,07 \mathbf{a}$ & $5,62 \pm 0,15 \mathbf{a}$ \\
$\mathbf{Z S}$ & $5,9 \pm 0,27 \mathbf{a}$ & $5,58 \pm 0,49 \mathbf{a}$ & $6,1 \pm 0,10 \mathbf{a}$ & $5,61 \pm 0,11 \mathbf{a}$ \\
\hline
\end{tabular}

Sites de prélèvement

\begin{tabular}{lll}
\hline Poquet & $5,71 \pm 0,12 \mathbf{a}$ & $5,78 \pm 0,09 \mathbf{a}$ \\
Interpoquet & $5,44 \pm 0,12 \mathbf{b}$ & $5,54 \pm 0,10 \mathbf{b}$ \\
\hline
\end{tabular}

\section{Cultures}

\begin{tabular}{ll}
\hline Niébé & $5,57 \pm 0,01 \mathbf{a}$ \\
Sorgho & $5,66 \pm 0,01 \mathbf{a}$ \\
\hline
\end{tabular}

NB : Dans une même colonne, les valeurs affectées de la même lettre ne sont pas significativement différentes selon le test de Newman-Keuls (SNK) au seuil de probabilité de 5\%.

$\mathrm{TA}=$ Témoin absolu, $Z \mathrm{C}=Z a \ddot{i}+$ compost, $Z \mathrm{CP}=Z a \ddot{i}+$ compost + Burkina phosphate

$\mathrm{ZF}=Z a \ddot{l}+$ fumier,$\quad \mathrm{ZFP}=Z a \ddot{\imath}+$ fumier + Burkina phosphate,$\quad \mathrm{ZS}=$ Zaï simple. 
Tableau 3 : Teneurs en carbone du sol (\%).

\begin{tabular}{lcccc}
\hline \multirow{2}{*}{ Traitements } & \multicolumn{2}{c}{ Niébé } & \multicolumn{2}{c}{ Sorgho } \\
\cline { 2 - 5 } & C.poquet & C.interpoquet & C.poquet & C.interpoquet \\
\hline TA & $1,19 \pm 0,12 \mathbf{a b}$ & $1,19 \pm 0,12 \mathbf{a}$ & $1,04 \pm 0,14 \mathbf{b c}$ & $1,04 \pm 0,14 \mathbf{a}$ \\
$\mathbf{Z C}$ & $1,20 \pm 0,06 \mathbf{a b}$ & $1,25 \pm 0,36 \mathbf{a}$ & $1,17 \pm 0,13 \mathbf{a b c}$ & $1,12 \pm 0,08 \mathbf{a}$ \\
$\mathbf{Z C P}$ & $1,21 \pm 0,12 \mathbf{a b}$ & $1,01 \pm 0,12 \mathbf{a}$ & $1,16 \pm 0,09 \mathbf{a b c}$ & $1,06 \pm 0,20 \mathbf{a}$ \\
$\mathbf{Z F}$ & $1,38 \pm 0,11 \mathbf{a}$ & $0,97 \pm 0,25 \mathbf{a}$ & $1,47 \pm 0,04 \mathbf{a}$ & $1,07 \pm 0,09 \mathbf{a}$ \\
$\mathbf{Z F P}$ & $1,34 \pm 0,07 \mathbf{a}$ & $1,21 \pm 0,21 \mathbf{a}$ & $1,30 \pm 0,22 \mathbf{a b}$ & $1,09 \pm 0,12 \mathbf{a}$ \\
$\mathbf{Z S}$ & $1,01 \pm 0,02 \mathbf{b}$ & $1,04 \pm 0,01 \mathbf{a}$ & $0,93 \pm 0,12 \mathbf{c}$ & $1,03 \pm 0,05 \mathbf{a}$ \\
\hline \multicolumn{5}{c}{ sites de prélèvement } \\
\hline Poquet & $1,22 \pm 0,04 \mathbf{a}$ & \multicolumn{3}{c}{$1,18 \pm 0,06 \mathbf{a}$} \\
Interpoquet & $1,11 \pm 0,12 \mathbf{b}$ & \multicolumn{3}{c}{$1,07 \pm 0,05 \mathbf{~ b}$} \\
\hline
\end{tabular}

\begin{tabular}{ll}
\hline & Cultures \\
\hline Niébé & $1,12 \pm 0,06 \mathbf{a}$ \\
Sorgho & $1,17 \pm 0,01$ a \\
\hline NB $:$ Dans une même colonne, les valeurs affectées de la même lettre ne sont pas significativement différentes selon le test de \\
Newman-Keuls (SNK) au seuil de probabilité de $5 \%$. \\
TA = Témoin absolu, ZC $=$ Zaï + compost, ZCP $=$ Zaï + compost + Burkina phosphate \\
ZF = Zaï + fumier, ZFP = Zaï + fumier + Burkina phosphate, ZS = Zaï simple.
\end{tabular}

Tableau 4 : Teneurs en azote (\%) du sol.

\begin{tabular}{lcccc}
\hline \multirow{2}{*}{ Traitements } & \multicolumn{2}{c}{ Niébé } & \multicolumn{2}{c}{ Sorgho } \\
\cline { 2 - 5 } & N poquets & N interpoquets & N poquets & N interpoquets \\
\hline TA & $0,092 \pm 0,007 \mathbf{a}$ & $0,092 \pm 0,007 \mathbf{a}$ & $0,080 \pm 0,008 \mathbf{b c}$ & $0,080 \pm 0,008 \mathbf{a}$ \\
ZC & $0,093 \pm 0,009 \mathbf{a}$ & $0,090 \pm 0,032 \mathbf{a}$ & $0,081 \pm 0,010 \mathbf{b c}$ & $0,074 \pm 0,004 \mathbf{a}$ \\
ZCP & $0,092 \pm 0,011 \mathbf{a}$ & $0,072 \pm 0,007 \mathbf{a}$ & $0,082 \pm 0,009 \mathbf{b c}$ & $0,072 \pm 0,014 \mathbf{a}$ \\
ZF & $0,110 \pm 0,010 \mathbf{a}$ & $0,075 \pm 0,012 \mathbf{a}$ & $0,111 \pm 0,009 \mathbf{a}$ & $0,075 \pm 0,009 \mathbf{a}$ \\
ZFP & $0,105 \pm 0,008 \mathbf{a}$ & $0,079 \pm 0,005 \mathbf{a}$ & $0,098 \pm 0,014 \mathbf{a b}$ & $0,075 \pm 0,008 \mathbf{a}$ \\
ZS & $0,070 \pm 0,003 \mathbf{b}$ & $0,071 \pm 0,005 \mathbf{a}$ & $0,065 \pm 0,008 \mathbf{c}$ & $0,070 \pm 0,005 \mathbf{a}$ \\
\hline
\end{tabular}

Sites de prélèvement

\begin{tabular}{lcc}
\hline Poquet & $0,094 \pm 0,003 \mathbf{a}$ & $0,086 \pm 0,002 \mathbf{a}$ \\
Interpoquet & $0,08 \pm 0,010 \mathbf{b}$ & $0,074 \pm 0,003 \mathbf{b}$ \\
\hline
\end{tabular}

\begin{tabular}{ll}
\hline & Cultures \\
\hline Niébé & $0,087 \pm 0,005$ a \\
Sorgho & $0,080 \pm 0,001 \mathbf{b}$ \\
\hline
\end{tabular}

NB : Dans une même colonne, les valeurs affectées de la même ne sont pas significativement différentes selon le test de Newman-Keuls (SNK) au seuil de probabilité de $5 \%$.

$\mathrm{TA}=$ Témoin absolu, $Z \mathrm{ZC}=Z a \ddot{i}+$ compost, $\mathrm{ZCP}=Z a \ddot{~}+$ compost + Burkina phosphate $\mathrm{ZF}=Z a \ddot{i}+$ fumier, $\mathrm{ZFP}=Z a \ddot{a}+$ fumier + Burkina phosphate, $Z \mathrm{~S}=$ Zaï simple. 
Tableau 5 : Taux de récupération après fractionnement.

\begin{tabular}{|c|c|c|c|c|c|}
\hline Cultures & Traitements & $\begin{array}{c}\text { Fraction A } \\
(\%) \\
\end{array}$ & $\begin{array}{c}\text { Fraction B } \\
(\%) \\
\end{array}$ & $\begin{array}{c}\text { Fraction C } \\
(\%) \\
\end{array}$ & Taux de récupération (\%) \\
\hline & TA & 52,34 & 22,05 & 25,76 & 100,15 \\
\hline & $\mathbf{Z F}$ & 38,13 & 27,91 & 34,77 & 100,81 \\
\hline & ZFP & 40,35 & 27,55 & 33,11 & 101,00 \\
\hline Niébé & $\mathbf{Z S}$ & 37,01 & 31,26 & 32,52 & 100,79 \\
\hline & TA & 43,03 & 24,63 & 32,67 & 100,33 \\
\hline & $\mathbf{Z F}$ & 38,87 & 31,23 & 30,10 & 100,20 \\
\hline & ZFP & 39,19 & 24,61 & 36,30 & 100,10 \\
\hline Sorgho & $\mathbf{Z S}$ & 35,51 & 31,88 & 32,33 & 99,72 \\
\hline
\end{tabular}

Tableau 6 : Teneurs en carbone et en azote $(\mathrm{g} / \mathrm{kg})$ des fractions.

\begin{tabular}{lccccccccccc}
\hline & & \multicolumn{3}{c}{ Fraction A } & \multicolumn{3}{c}{ Fraction B } & \multicolumn{3}{c}{ Fraction C } \\
\cline { 3 - 10 } Cultures & Traitements & $\mathbf{N}$ total & $\mathbf{C}$ total & $\mathbf{C} / \mathbf{N}$ & $\mathbf{N}$ total & $\mathbf{C}$ total & $\mathbf{C} / \mathbf{N}$ & $\mathbf{N}$ total & $\mathbf{C}$ total & $\mathbf{C} / \mathbf{N}$ \\
\hline Niébé & TA & 2,17 & 20,99 & 9,7 & 0,27 & 4,75 & 17,8 & 0,4 & 7,32 & 18,5 \\
& ZF & 2,18 & 19,75 & 9 & 0,33 & 4,21 & 12,9 & 1,17 & 14,99 & 12,8 \\
& ZFP & 2,07 & 19,21 & 9,3 & 0,38 & 5,04 & 13,2 & 1,28 & 16,43 & 12,8 \\
& ZS & 2,02 & 19,15 & 9,5 & 0,23 & 4,14 & 17,7 & 0,38 & 6,72 & 17,8 \\
\hline \multirow{2}{*}{ Sorgho } & TA & 2,00 & 18,81 & 9,4 & 0,33 & 5,75 & 17,2 & 0,26 & 5,27 & 20,5 \\
& ZF & 2,13 & 20,6 & 9,7 & 0,37 & 4,96 & 13,5 & 1,24 & 17,54 & 14,1 \\
& ZFP & 2,08 & 20,16 & 9,7 & 0,25 & 3,26 & 12,9 & 1,09 & 15,33 & 14,1 \\
& ZS & 1,88 & 19,31 & 10,3 & 0,17 & 2,65 & 15,6 & 0,36 & 6,98 & 19,2 \\
\hline
\end{tabular}

TA : Témoin absolu ZF : Zaï+ fumier $\quad$ ZFP : Zaï+ fumier + Burkina phosphate $\quad$ ZS : Zaï simple

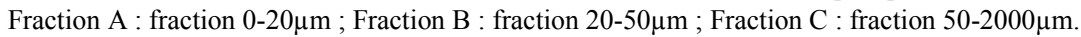




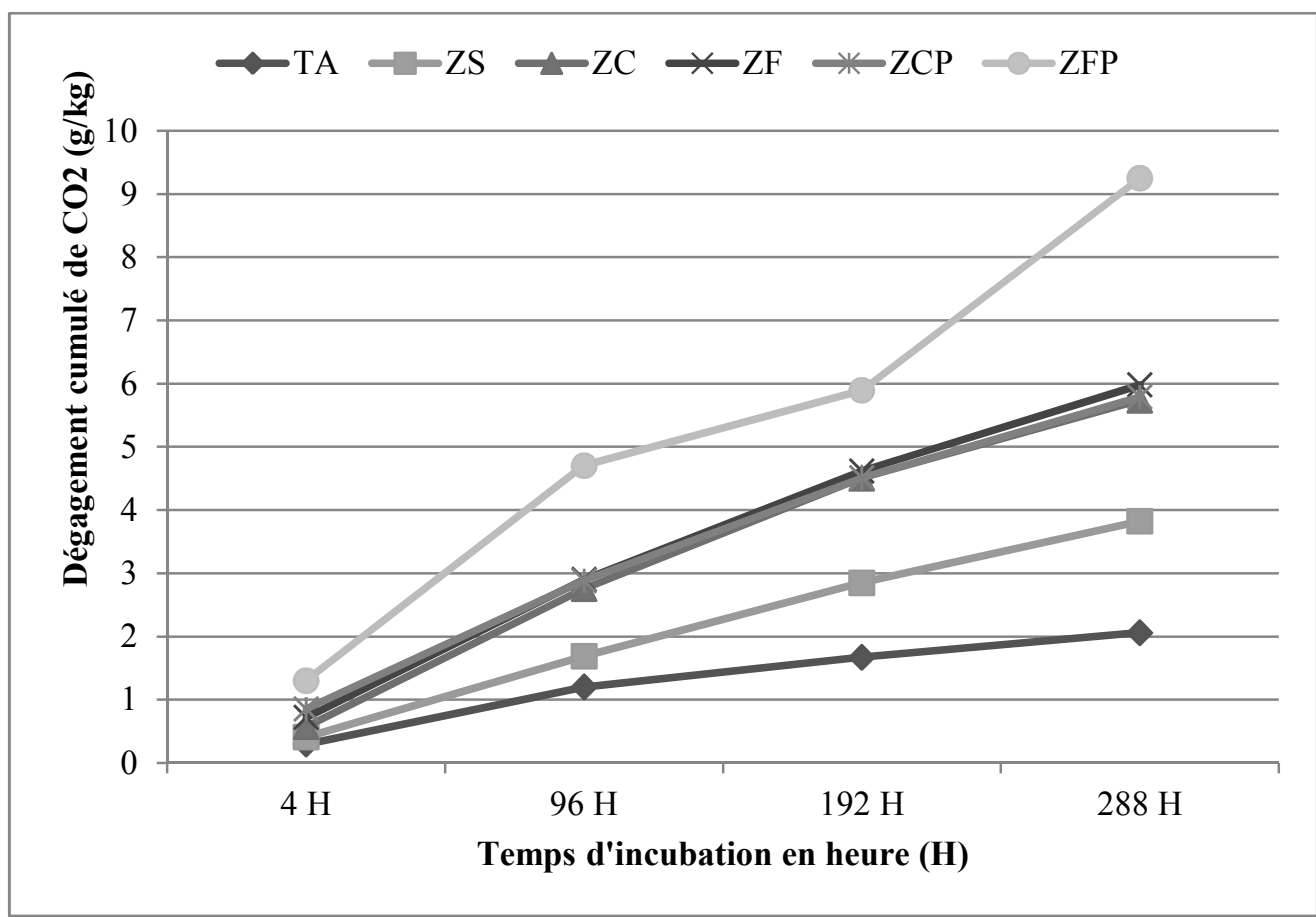

Figure 1 : Respiration du sol sous sorgho.

$\mathrm{TA}=$ Témoin absolu, $\mathrm{ZC}=Z a \ddot{~}+$ compost, $\mathrm{ZCP}=Z a \ddot{i}+$ compost + Burkina phosphate,

$\mathrm{ZF}=Z a \ddot{ }+$ fumier, $\mathrm{ZFP}=Z a \ddot{i}+$ fumier + Burkina phosphate, $\mathrm{ZS}=$ Zaï simple.

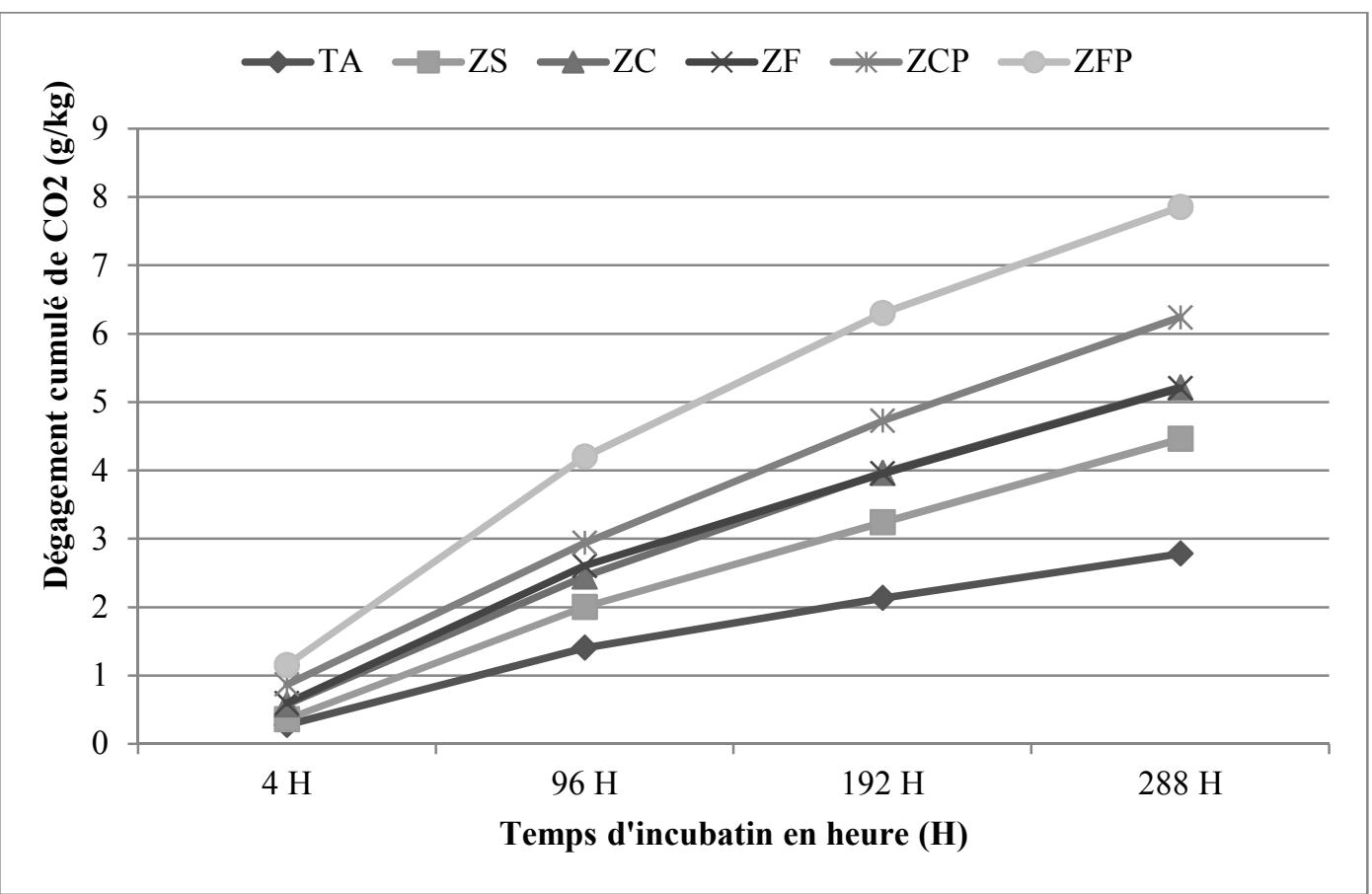

Figure 2 : Respiration du sol sous niébé.

$\mathrm{TA}=$ Témoin absolu, $\mathrm{ZC}=Z a \ddot{i}+$ compost, $\mathrm{ZCP}=Z a \ddot{i}+$ compost + Burkina phosphate, $\mathrm{ZF}=Z a \ddot{i}+$ fumier, $\mathrm{ZFP}=$ Zä̈ + fumier + Burkina phosphate, $\mathrm{ZS}=$ Zaï simple. 


\section{DISCUSSION}

\section{Effets des pratiques sur le $\mathbf{p H}_{\text {eau }}$ du sol}

La récupération du zipella par le zaï a permis de relever significativement le $\mathrm{pH}$ du sol. Ce relèvement du $\mathrm{pH}$ s'accroit avec l'apport de la matière organique principalement le compost combiné au Burkina phosphate. En effet, les poquets sont des cuvettes qui permettent de collecter les eaux de ruissellement. Ce rôle bénéfique du zaï couplé au pouvoir stabilisateur de la matière organique en particulier le compost, serait amélioré par l'application du Burkina phosphate. Ainsi, cette baisse d'acidité serait liée au calcium contenu dans ce phosphate naturel qui, en se liant au complexe argilohumique permettrait de baisser la concentration des ions $\mathrm{H}^{+}$dans la solution du sol. Les résultats de cette étude sont corroborés par ceux obtenus par Maré (2009), qui ont montré que les $\mathrm{pH}$ des interpoquets se situent entre 5 et 5,8 tandis que ceux des poquets sont compris entre 5,7 à 6,7 .

\section{Effets des pratiques sur les teneurs en carbone et en azote}

L'effet des apports de fumures organominérales sur le relèvement des taux de carbone et d'azote du sol est avéré (Somé, 2015 ; Ouandoago et al., 2016). L'évolution positive et significative des taux de carbone et d'azote dans les poquets de zaï comparativement aux interpoquets illustre bien l'impact bénéfique de cette technique de restauration dans la récupération des sols. Le zaï en relevant le $\mathrm{pH}$ dans les poquets de zipellé, aurait créé une bonne condition d'évolution du compost et du fumier apportés via l'amélioration de l'activité biologique. Ce qui a favorisé le relèvement significatif des taux de carbone et d'azote du sol dans les poquets surtout lorsqu'ils ont été amendés avec le fumier. Les poquets constituent en outre des cuvettes de rétention d'éléments fins et de matières organiques et par conséquent constitueraient des îlots d'éléments nutritifs. Cette dégradation de la matière organique par les organismes décomposeurs semble limitée sur les interpoquets où la croûte de battance constitue un obstacle à l'infiltration des eaux de pluie et à l'aération du sol. A ce titre, Somé et al. (2004) concluaient que le zaï permettait d'améliorer l'infiltration et la rétention d'eau. Ces résultats, conformes à ceux de Maré (2009), confirment le rôle protecteur et de maintien des nutriments des poquets de zaï contre les effets du vent et de l'eau sur les zipella en particulier. La contribution du zaï à l'enrichissement des sols s'améliore surtout quand il est associé à un amendement. En absence d'amendement ou de fertilisant, un appauvrissement en $\mathrm{C}$ et $\mathrm{N}$ pourrait être observé du fait des exportations. Dans notre étude, les teneurs en $\mathrm{C}$ et $\mathrm{N}$ sous le témoin ont été supérieures à ZS. Rappelons que la productivité est nulle sous témoin. Dans les parcelles ayant produit de la biomasse, notamment ZS, La culture aurait favorisé l'exportation des éléments nutritifs dans la biomasse produite. Les études précédentes sur ce même essai ont montré que les teneurs en $\mathrm{C}$ et $\mathrm{N}$ étaient plus élevées sous zaï associé à l'apport du fumier combiné au Burkina phosphate (Maré, 2009; Dabré, 2011) ou à l'urée (Zongo, 2013). Cela peut être lié à la fertilité chimique du fumier utilisé ou à une amélioration des exportations dues à la présence du phosphore. En général, lorsque le sol présente un certain équilibre nutritionnel, la nutrition des plantes est améliorée, partant les exportations. Il est maintenant bien établi, que pour maintenir la fertilité des sols de nos agroécosystèmes, il faut $\mathrm{y}$ retourner sous forme de compost ou de fumier les résidus de récoltes. En outre, le niébé permet de rehausser le taux d'azote dans le sol par rapport au sorgho quand les poquets sont amendés avec le fumier. Le niébé étant une légumineuse, a une source supplémentaire d'azote que le sorgho : la fixation symbiotique de l'azote atmosphérique. Le niébé peut donc 
permettre d'améliorer le statut de l'azote du sol. Toutefois, l'addition du phosphore au fumier et au compost n'a pas favorisé l'accroissement de la teneur en azote malgré son impact sur le bon développement racinaire. Il est bien connu que le phosphore favorise la nodulation du niébé mais sa capacité à fixer l'azote atmosphérique dépend également de plusieurs paramètres dont le $\mathrm{pH}$, l'état hydrique du sol, le taux initial d'azote du sol et le potentiel des rhizobia. En effet, l'application de doses croissantes de phosphore augmente la production de biomasse nodulaire et par conséquent la fixation symbiotique chez le niébé (Jemo et al., 2006). Inversement, une bonne nutrition en azote favorise l'absorption du phosphore, expliquant l'effet bénéfique des légumineuses sur la nutrition phosphatée. L'essai est conduit depuis 2006 et soumis aux mêmes traitements chaque année. Au fil du temps, la solubilisation progressive du phosphate naturel apporté a pu conduire à des doses limitant son impact sur la nodulation et la fixation d'azote atmosphérique.

\section{Effets des pratiques sur la répartition et la qualité de la matière organique}

L'étude a montré que la grande partie de la matière organique contenue dans les poquets de zaï est localisée dans la fraction fine du sol. Ces résultats sont similaires à ceux déjà observés par Somé et al. (2015) dans le même agrosystème. Cette matière organique est liée aux particules fines (limons fins, argiles) donc séquestrée durablement dans le sol. Cela constitue à n'en point douter une alternative pour limiter les émissions de gaz à effet de serre permettant de diminuer la part de l'agriculture conventionnelle dans ces émissions. De par son rapport $\mathrm{C} / \mathrm{N}$ très faible, cette fraction est riche en azote. Cet azote est corrélé à la teneur du sol en éléments fins (argile et limons fins) comme le souligne Dridi et Gallali (2006). Il est généralement localisé dans l'humine (Dridi et Gallali, 2006).
Il est aussi très protégé des phénomènes de dégradation (chimique, biochimique) du fait de son association intime avec les limons et les argiles et de sa composition intrinsèque qui la rend résistante. La matière organique la plus sensible aux pratiques agricoles avec un turn-over rapide (2 à 3 ans) dite libre a un faible taux et est stocké dans la fraction 50$2000 \mu \mathrm{m}$. Les résultats de cette étude confirment ceux de certains auteurs selon lesquels l'argile et le limon fin favorisent l'accumulation de la matière organique sous forme de complexe organo-minéraux stables (Baldock et Skjemstad, 2000; Feller et al., 2001 ; Barthès et al., 2008). Mais, les travaux de Christensen (2001) ont prouvé qu'en plus des teneurs en azote, les teneurs en carbone sont aussi plus élevées dans cette fraction quels que soit les traitements. Constituée de 50 à $90 \%$ de l'azote de la fraction fine, l'humine est la réserve la plus importante de cette fraction du sol selon les mêmes auteurs. Il ressort de l'étude que la représentativité de cet azote de la fraction argilo-limoneuse est inférieure aux résultats de Dridi et Gallali (2006), une différence qui s'explique par la texture $\mathrm{du}$ sol utilisé. Pour des sols très pauvres surtout en azote et en phosphore, il paraît logique que leur fraction fine soit moins riche en azote. Etant très stable, l'azote de la fraction fine est faiblement influencé par la nature de la culture mise en place.

\section{Effet des pratiques sur le dégagement de $\mathrm{CO}_{2}$ du sol}

Le dégagement important de $\mathrm{CO}_{2}$ obtenu surtout dans les poquets de zaï amendés avec du fumier associé au Burkina phosphate s'explique par l'augmentation des teneurs en matière organique et le relèvement de $\mathrm{pH}$ avec ce traitement. Le $\mathrm{pH}$ et la matière organique influencent le potentiel d'activité biologique du sol. On sait par ailleurs que le travail du sol (ameublissement du sol dans les poquets dans le cas présent) entraine une baisse de la densité apparente du sol (Sermé et 
al., 2015) se traduisant par une amélioration de l'humidité et de l'aération du sol. Dans ces conditions, les microorganismes se développent et induisent une minéralisation accélérée de la matière organique en présence dont la quantité de $\mathrm{CO}_{2}$ produite est fonction de la population microbienne, leur diversité, les enzymes métaboliques secrétées et la composition des amendements appliqués. Ces conclusions s'inscrivent dans la même logique de plusieurs auteurs qui ont montré que la respiration du sol est effectivement fonction de la température du sol, de l'humidité (Perrin et al., 2004 ; Sandor, 2010), de la texture, du $\mathrm{pH}$ et des teneurs totales en carbone et en azote (Wang et al., 2006 ; Gnankambary et al., 2008). La fertilité des sols du Burkina Faso dans leur grande majorité étant limitée par l'azote et le phosphore; la présence de ces éléments dans le traitement ZFP a permis d'accroître l'activité biologique potentielle globale. L'apport combiné du Burkina phosphate et le fumier ou le compost a engendré une augmentation du dégagement de $\mathrm{CO}_{2}$ respectivement $34,61 \%$ et à $9 \%$ par rapport au zaï amendé uniquement au fumier ou au compost. Ce résultat confirme ceux de travaux similaires qui relèvent que le phosphore est l'élément le plus indispensable à l'efficacité de l'activité microbienne du sol (Gnankambary et al., 2008 ; Diarra, 2009) sur les sols tropicaux du fait de leur pauvreté en phosphore (Compaoré et al., 2004). Cette étude montre que le sorgho favorise une relative baisse d'acidité par rapport au niébé. Cela induit probablement une activité biologique globale plus avantageuse sous cette culture même si la différence n'est pas significative. De plus, le compost semble avoir des effets stimulateurs de l'activité microbienne du sol moins intenses que les déchets organiques non compostés soit à cause de la qualité du compost utilisé, soit du fait de la réduction de leur concentration en substances métaboliques (Emmerling et al., 2000).

\section{Conclusion}

Les résultats de cette étude montrent que les zipella peuvent être restaurés par le zaï associé à des apports organiques et minéraux. Cette technique améliore significativement les paramètres chimiques $(\mathrm{pH}, \mathrm{C}$ et $\mathrm{N})$ du fait de la concentration dans les poquets de zaï de la matière organique et des éléments fins. L'amendement des poquets de zaï avec le fumier associé au phosphate a contribué à accroître significativement l'activité biologique globale. Le relèvement des teneurs en $\mathrm{C}$ et $\mathrm{N}$ peut être toutefois limité par l'accroissement des exportations en lien avec la production en biomasse élevée des cultures lorsque le fumier est combiné au Burkina phosphate dans les poquets. A l'exception de l'azote, l'étude a montré que l'espèce végétale n'influence pas significativement les paramètres chimiques, l'activité biologique potentielle et la qualité de la matière organique du sol si les résidus de cultures sont totalement exportés. Elle montre par ailleurs que l'essentiel de la matière organique du sol est préférentiellement liée aux limons fins et aux argiles et donc difficilement dégradable. Le zaï associé à des apports organiques et minéraux semble être une pratique pertinente de séquestration de $\mathrm{C}$ et $\mathrm{N}$ dans les agroécosystèmes sub-sahéliens dans un contexte de changement globaux.

\section{CONFLIT D'INTERETS}

Les auteurs déclarent qu'il n'y pas de conflit d'intérêts entre eux.

\section{CONTRIBUTIONS DES AUTEURS}

$\mathrm{EH}$ a assuré la direction scientifique de ce travail de bout en bout. Le travail de correction du manuscrit a été également assuré par lui. Il est d'ailleurs l'auteur correspondant de cet article. DS a participé à la correction du présent manuscrit soumis à 
votre journal pour publication. JJD a participé à la correction du présent manuscrit.

\section{REMERCIEMENTS}

Les auteurs témoignent leur gratitude à Moussa BARRY, Prosper SAWADAGO, techniciens au Laboratoire d'Agro-écologie, sur le centre IRD de Ouagadougou.

\section{REFERENCES}

Baldock JA, Skjemstad JO. 2000. Role of the soil matrix and minerals in protecting natural organic materials against biological attack. Organic Geochemistry, 31(7): $697-$ 710. DOI: 10.1016/S0146-6380(00)000498

Barthès BG, Kouakoua E, Larré-Larrouy MC, Razafimbelo TM, De Luca EF, Azontonde A, Neves CSVJ, De Freitas PL, Feller CL. 2008. Texture and sesquioxide effects on water-stable aggregates and organic matter in some tropical soils. Geoderma, 143: 1425. DOI: $10.1016 /$ j.geoderma.2007.10.003

Barro A, Zougmoré R, Taonda JBS. 2005. Mécanisation de la technique du zai manuel en zone semi-aride. Cahiers Agricultures, 14(6): 549-559. http://revues. cirad.fr/index.php/cahiersagricultures/article/view/30550/30310

Christensen BT. 2001. Physical fractionation of soil and structural functional complexity in organic matter turnover. Eur. J. Soil Sci., 52: 345-353. DOI: 10.1046/j.1365-2389.2001.00417.x

Compaoré E, Fardeau JC, Morel JL, Sedogo MP. 2001. Le phosphore biodisponible des sols : une des clés de l'agriculture durable en Afrique de l'Ouest. Cah. Agric., 10: 75142.

http://revues.cirad.fr/index.php/cahiersagricultures/article/view/30283

CPCS. 1967. Classification des sols. Commission de Pédologie et de Cartographie des Sols, ENSA-Grignon, Laboratoire de Pédologie-Géologie, Paris, France.
Dabat MH, Lahmar R, Guissou R. 2012. La culture du niébé au Burkina Faso : une voie d'adaptation de la petite agriculture à son environnement ? Autrepart, 62: 95114. DOI: $10.3917 /$ autr.062.0095

Dabré A. 2011. Efficience du Burkina phosphate et des amendements organiques sur la production végétale et la dynamique de l'activité biologique des sols très dégradés (zipella) du Burkina Faso : Cas $\mathrm{du}$ village de Pougyango (Province du Passoré). Rapport de stage de fin de cycle. BTS/Pédologie, CAP/M, Bobo-Dioulasso, $84 \mathrm{p}$.

Diarra BG. 2009. Influence du phosphore, de l'azote et du houppier sur les rendements $\mathrm{du}$ sorgho (Sorghum bicolor), les fractions $\mathrm{du}$ phosphore ct l'activité des microorganismes du sol d'un parc agroforestier de la zone soudanienne du Burkina Faso. Mémoire de fin de cycle d'Ingénieur du Développement Rural option Agronomie, IDR, Université Polytechnique de Bobo-Dioulasso, Institut du Développement Rural Burkina Faso, $70 \mathrm{p}$.

Dridi I, Gallali T. 2006. Distribution de l'Azote et caractérisation des sols de la Tunisie du Nord. Géo-Eco-Trop., 30(2): 87-96.

http://geoecotrop.be/uploads/publications/ pub_302_504821

Emmerling C, Liebner C, Haubold-Rosar M, Katzur J, Schroder D. 2000. Impact of application of organic waste materials on microbial and enzyme activities of mine soils in the Lusatian coal mining region. Plant Soil, 220: 129-138. DOI: 10.1023/A:1004784525209

Feller C. 1979. Une méthode de fractionnement granulométrique de la matière organique des sols. Application aux sols tropicaux, à texture grossière, très pauvres en humus. Cah. ORSTOM, Série Pédol., 17(4): 339-346. 
Feller C, Balesdent J, Nicolardot B, Cerri C. 2001. Approaching "Functional" Soil Organic Matter Pools through ParticleSize Fractionation: Examples for Tropical Soils. In Assessment Methods for Soil Carbon, Lal R, Kimble JM, Follet RF, Stewart BA (eds). CRC Press LLC, Lewis Publishers; 53-67.

Gnankambary Z, Ilstedt U, Nyberg G, Hien V, Malmer A. 2008. Nitrogen and phosphorus limitation of soil respiration in two tropical agroforestry park lands in the south-

Sudanese zone of Burkina Faso: the effects of tree canopy and fertilization. Soil

Biology and Biochemistry, 40: 350-359.

DOI:

http://dx.doi.org/10.1016/j. soilbio.2007.08 .015

Jemo M, Abaidoo RC, Nolte C, Tchienkoua M, Sanginga N, Horst WJ. 2006. Phosphorus benefits from grain-legume crops to subsequent maize grown on acid soils of southern Cameroon. Plant Soil, 284: 385-397. DOI: http://dx.doi.org/10.1007/s11104-0060052-x

Mando A, Zougmoré R, Zombré N. P, Hien V. 2001. Réhabilitation des sols dégradés dans les zones semi-arides de l'Afrique sub-sahélienne. In La Jachère en Afrique Tropicale (Vol.II), Floret C, Pontanier R (eds). John Libbey Eurotext: Paris ; 311339.

Maré BT. 2009. Impact de l'utilisation du niébé et de divers amendements sous zaï sur les caractéristiques des sols dégradés : Conséquences pédo-socio-économiques. Mémoire de fin de cycle, Diplôme d'ingénieur de conception en vulgarisation agricole, IDR, Université Polytechnique de Bobo Dioulasso, 61p.

MARH. 2008. Evolution du secteur agricole et des conditions des ménages au Burkina Faso. Projet AI/CN-SISA. 92p.

Naab JB, Chimphango SMB, Dakora FD. 2009. $\mathrm{N}_{2}$ fixation in cowpea plants grown in farmers' fields in the Upper West Region of Ghana, measured using ${ }^{15} \mathrm{~N}$ natural abundance. Symbiosis, 48: 37-46. DOI: 10.1007/BF03179983

Ouandaogo N, Ouattara B, Pouya BM, Gnankambary Z, Nacro BH, Sedogo PM. 2016. Effets des fumures organo-minérales et des rotations culturales sur la qualité des sols. Int. J. Biol. Chem. Sci., 10(2): 904918. DOI http://dx.doi.org/10.4314/ijbcs.v10i2.37

Perrin D, Laitat E, Yernaux M, Abinet M. 2004. Modélisation de la réponse des flux de respiration d'un sol forestier selon les principales variables climatiques. Biotechnol. Agron. Soc. Environ., 8(1): 1525.

Sandor M. 2010. Soil respiration: Concept and Measurement Methods. Pro Environment, 3: 54-57.

Sawadogo H. 2006. Fertilisation organique et phosphatée en système de culture zaï en milieu soudano-sahélien du Burkina Faso. Thèse de doctorat. Faculté Universitaire des Sciences Agronomiques de Gembloux, Belgique, 232p.

SP/CONEDD. 2006. Revue scientifique sur l'état de la dégradation des terres au Burkina Faso, Rapport final. MECV, Ouagadougou, Burkina Faso, 105p.

Sawadogo H, Bock L, Lacroix D, Zombré NP. 2008. Restauration des sols dégradés à l'aide du zaï et du compost dans le Yatenga (Burkina Faso). Biotechnol. Agron. Soc. Environ., 12(3): 279-290. http://popups.ulg.ac.be/17804507/index.php?id=2573

Sermé I, Ouattara K, Logah V, Taonda JB, Pale S, Quansah C, Abaidoo CR. 2015. Impact of tillage and fertility management options on selected soil physical properties and sorghum yield. Int. J. Biol. Chem. Sci., 9(3): $\quad 1154-1170 . \quad$ DOI http://dx.doi.org/10.4314/ijbcs.v9i3.2

Somé D. 2015. Contribution des légumineuses dans le système zaï céréalier à la 
réhabilitation des sols dégradés (Zipella) et à la production : Cas du niébé (Vigna unguiculata (L.) Walp.) sur sol ferrugineux tropical dans la Région Nord du Burkina Faso. Thèse de doctorat, Université de Ouagadougou, Burkina Faso. 126p.

Somé D, Hien E, Assigbetse K, Drevon JJ, Masse D. 2015. Dynamique des compartiments du carbone et de l'azote dans le sol cultivé en niébé et sorgho dans le système zaï en zone Nord soudanienne du Burkina Faso. Int. J. Biol. Chem. Sci., 9(2): 954-969. DOI: http://dx.doi.org/10.4314/ijbcs.v9i2.32

Somé D, Zombré PN, Zombré G, Macauley HR. 2004. Impact de la technique du zaï sur la production du niébé et sur l'évolution des caractéristiques chimiques des sols très dégradés (zipellé) du Burkina Faso. Sécheresse, 15(3): 263-269.

Traoré K, Toé AM. 2008. Capitalisation des initiatives sur les bonnes pratiques agricoles au Burkina Faso. DVRD/DPV/MAHRH, Ouagadougou, Burkina Faso. 99p.

Traoré OYA. 2012. Etude de la fixation symbiotique de l'azote atmosphérique par le niébé (Vigna unguiculata L. Walp) avec la méthode de l'abondance naturelle en ${ }^{15} \mathrm{~N}$ sous diverses pratiques culturales dans le Centre-Ouest du Burkina Faso. Mémoire de DEA en Gestion Intégrée des Ressources Naturelles, Sciences du sol. IDR, Université Polytechnique de Bobo, Burkina Faso, 53p.

Wang W, Guo JX, Feng F, Oikawa T. 2006.

Contribution of root respiration to total soil respiration in a Leymus chinensis (Trin.) Tzvel. Grassland of Northeast China. Journal of Integrative Plant Biology, 48(4): 409-414. DOI: 10.1111/j.1744-7909.2006.00241.x

Zeinabou H, Mahamane S, Bismarck NH, Bado BV, Lompo F, Bationo A. 2014. Effet de la combinaison des fumures organo-minérales et de la rotation niébémil sur la nutrition azotée et les rendements du mil au sahel. Int. J. Biol. Chem. Sci., 8(4): 1620-1632. DOI : http://dx.doi.org/10.4314/ijbcs.v8i4.24

Zombré NP. 2003. Les sols très dégradés «zipella » du centre nord $\mathrm{du}$ Burkina Faso: dynamique, caractérisations morpho-bio-pédologiques et impacts des techniques de restauration sur leur productivité. Thèse de doctorat d'Etat es Sciences naturelles, Université de Ouagadougou, Burkina Faso, 326p.

Zombré NP. 2006. Evolution de l'occupation des terres et localisation des sols nus dans le Centre Nord du Burkina Faso. Télédétection, 5(4): 285-297.

Zongo KF. 2013. Associations légumineusescéréales dans les agrosystèmes soudanosahéliennes du Burkina Faso : Perceptions et pratiques paysannes, effets du zaï et des amendements organiques et organominéraux sur les rendements des cultures associées niébé-sorgho. Mémoire de DEA en Science du sol. IDR, Université Polytechnique de Bobo, Burkina Faso, $68 \mathrm{p}$. 